\title{
Evaluation of E492 Microcin Gene Presence in Klebsiella pneumoniae Collected from Patients Registered to Educational Hospitals of Isfahan
}

\author{
Maryam Nasresfahani, ${ }^{1, *}$ Ali Mohammad Ahadi, ${ }^{2}$ Hoda Ayat, ${ }^{2}$ and Hashem Nayeri ${ }^{3}$ \\ ${ }^{1}$ Department of Microbiology, Falavarjan Branch, Islamic Azad University, Isfahan, Iran \\ ${ }^{2}$ Department of Genetics, Faculty of Science, Shahr-e-Kord University, Shahr-e-Kord, Iran \\ ${ }^{3}$ Department of Biochemistry, Falavarjan Branch, Islamic Azad University, Isfahan, Iran \\ "Corresponding author: Maryam Nasresfahani, MSc Student of Microbiology, Department of Microbiology, Falavarjan Branch, Islamic Azad University, Isfahan, Iran. E-mail: \\ m.esfahni66@gmail.com
}

Received 2015 December 13; Revised 2016 June 24; Accepted 2017 January 09.

\begin{abstract}
Background: Microcin E492 is a low molecular weight channel-forming microbial toxin that is produced by some Klebsiella pneumoniae strains. This peptide is active in some strains of Enterobacteriaceae family, and it has different applications in medicine.

Objectives: The present descriptive analytical study was carried out to determine the presence of Klebsiella pneumoniae with gene encoding Microcin E492 in clinical specimens isolated from patients registered in two hospitals in Isfahan, Iran.

Methods: In this interventional-descriptive study, 45 clinical specimens including 20 urine clinical specimens, 10 respiratory tract clinical specimens and 15 burn wounds specimens were selected from Al-Zahra and Imam Musa Kazem hospitals in Isfahan during the year 2013. Total DNA was extracted from clinical specimens and hot start PCR was optimized using specific primers for the amplification of the complete sequence of E492 gene. Fidelity of PCR products was confirmed by direct sequencing. Homology analysis was performed by application of BLAST serve. The data were analyzed with Chromasv2.1.1 software.

Results: In forty-five collected clinical specimens (20 urine clinical specimens, 10 respiratory tractclinical specimens and 15 burn wounds specimens), 20 samples (44.4\%) were screened with Klebsiella pneumoniae contamination. PCR analysis was showed presence of $\mathrm{E} 492$ gene in $40 \%$ ( 8 samples) of contaminated clinical specimens.

Conclusions: About $40 \%$ of the clinical specimens collected from Isfahan hospitals contaminated with Klebsiella pneumoniae had microcin E492 gene. These types of microcins are low molecular weight antibiotic peptides produced by Enterobacteriaceae with a wide range of antibacterial activity against Gram-negative bacteria. This property can be useful for antibacterial trials. Existence of microcin gene in Enterobacteriaceae can develop them into a dominant microbial flora in human body internal environment.

Keywords: Microcin E492, Bacteriocin, Klebsiella pneumoniae
\end{abstract}

\section{Background}

Klebsiella pneumoniae is an opportunistic pathogen commonly found in the environment and can colonize and infect both plants and animals. It is a leading cause of hospital-acquired infections. Widely, it can be carried asymptomatically in the intestinal tract, skin, nose, and throat of healthy individuals, but it can also cause a range of infections in hospitalized patients, most commonly pneumonia, wound, soft tissue, or urinary tract infections. Its infections are particularly a problem among neonates, the elderly, and those with immunodeficiency $[1,2]$. Because they survive in the environment and their competition with other microorganisms for resources, bacteria produce antimicrobial compounds to inhibit or kill other competing strains. All bacteria produce some types of antimicrobial compounds, and we need to find the right indicator strains and growth conditions to diagnose them. The antimicrobial peptides are just one of the many classes of antimicrobial compounds. Based on their biosynthetic mechanisms, the antimicrobial peptides are divided into two groups, the bacteriocins and the antibiotic peptides. Bacteriocins are synthesized by ribosomes in bacteria that are active against closely related strains. One of these peptides, microcin E492, is a highly hydrophobic molecule with $5 \mathrm{kDa}$ molecular weight [3-5].

The bacteriocins produced by Gram-positive bacteria are divided into two groups based on molecular weight, the colicin-type (high molecular mass, 25-80 kDa) and the microcins (low molecular mass, $<10 \mathrm{kDa}$ ). The mechanisms wich those bacteriocins perform are diverse, and include pore-formation, DNAses, RNAses, inhibitition of protein synthesis or DNA replication, etc [6, 7].

Bacteriocins secreted by producer strain include varied classes of proteins with toxic effects on closely related gram negative strains. Microcins are presently divided into two classes. Class I comprises three plasmid-encoded peptides with a molecular weight under $5 \mathrm{kDa}$ that have widespread post-translational modifications: microcins J25, C (called C7, C51 or C7/C51), and B17. Class II microcins 
comprise higher molecular weight peptides in a range of 5 $-10 \mathrm{kDa}[8,9]$.

Microcin E492, produced by a strain of Klebsiella pneumoniae, has low-molecular-weight (7.887 Da) with channelforming features. This microcin is able to form amyloidlike fibrils that can be used as an anti tumoral agent. These fibrils can be used as stable sources to steadily release of biologically active molecules $[10,11]$. In the present study, this question is answered:

How many of Klebsiella pneumoniae strains isolated from hospitals contain microcin E492 and what is the frequency? In the present study, determination of the presence and detection of the gene encoding microcin E492 in Klebsiella pneumoniae strains isolated randomly from hospitals in Isfahan Province of Iran, were studied. This experimental study was done during the year 2013.

\section{Methods}

\subsection{Sampling and Bacteria Isolation}

This interventional-descriptive study was performed to evaluate the frequency of Klebsiella pneumoniae producing microcin E492 in clinical specimens of burn wounds, urine and respiratory tracts of patients registered in Isfahan hospitals. For this purpose, forty-five isolates of Klebsiella pneumoniae were recovered from burn wounds, urines, and respiratory tract of patients, 15, 20, 10 clinical specimens respectively, from patients by random. The isolates were identified by conventional biochemical and standard microbiology tests including colonial morphology (Eosin methylene blue, Macconkey agar 37c/24 hours) [12], TSI, SIM, and Simon citrate agar [13-15].

\subsection{DNA Extraction}

Total DNA was extracted from different cultured bacterial isolates by DNA extraction Talizol-D kit (Tali Gene Pars Company, Iran) according to the prepared manual. Extracted DNA was qualified by using $1.5 \%$ agarose gel electrophoresis.

\subsection{Amplification of Microcin E492 Gene}

A hot start polymerase chain reaction was optimized for screening of the microcin E492 gene using specific primers (Table 1) designed for gene encoding microcin E492 with access number "AF063590.3" in the national center for biotechnology information (NCBI) genome data bank. The primers were qualified and analyzed by using the Gene Runner v4.0 software [16, 17]. PCR reactions were carried out with $0.1 \mathrm{mg} / \mu \mathrm{L}$ of the DNA template, $0.4 \mathrm{Pm} / \mathrm{lit}$ of each primer (FMN and RMN), PCR buffer 1x, $200 \mu \mathrm{M}$ dNTP, 1/5 mM MgCl2 final concentrations and $1 \mathrm{U} / \mathrm{R}$ of Taq
DNA polymerase (Sinaclon co.) in a total volume of $25 \mu \mathrm{L}$ (Table 2). Amplification was carried out in a thermal cycler (Applied Biosystem, 2720) with the following thermal cycling conditions: 5 minutes at $94^{\circ} \mathrm{C}$ and 30 cycles of amplification consisting of 45 seconds at $94^{\circ} \mathrm{C}, 45$ seconds at $64^{\circ} \mathrm{C}$, and 30 seconds at $72^{\circ} \mathrm{C}$, with 5 minutes at $72^{\circ} \mathrm{C}$ for the final extension. PCR products were analyzed by electrophoresis on a $1 \%$ agarose gel at $85 \mathrm{~V}$ for 45 minutes. DNA bands visualized by green viewer (Merc) staining and following UV detection.

\subsection{Sequencing Method}

The PCR purification kit (Thermo scientific Co., USA) was used to purify PCR products, and sequencing was performed by Faza biotech company (Iran) using forward and reverse specific primers. The nucleotide sequences were analyzed with Chromasv2.1.1 software (http://technelysium.com.au/) and finally homology analysis was performed by application of BLAST server (http://www.ncbi.nlm.nih.gov/BLAST).

\section{Results}

Forty-five clinical specimens were collected from burn wound, urine, and respiratory tract of patients $(15,20$, and 10 , respectively) registered in hospitals in Isfahan, Iran. Of the 45 clinical specimens, 20 (44.4\%) contained Klebsiella pneumoniae.

\subsection{DNA Extraction from Klebsiella pneumoniae}

After the initial diagnosis of Klebsiella pneumoniae, genomic bacterial DNA was extracted and applied in a $1 \%$ agarose gel electrophoresis (Figure 1).

\subsection{Electrophoresis of PCR Products}

PCR products were run in $1 \%$ agarose gel electrophoresis. As shown in Figure 2A, the PCR products fragment of the amplicon have a size of $277 \mathrm{bp}$ (Figure 2). In order to purify the initial PCR product, DNA gel extraction kit (Fermentase \#K0512) was used, and the purified PCR product (Figure 2B) was sequenced.

\subsection{Sequencing}

In order to confirm the data, purified PCR products harbor microcin E492 were sequenced. The homology of nucleotide sequences was analyzed with Chromas v1.45 software and the BLAST server (http://www.ncbi.nlm.nih.gov/BLAST). 
Table 1. Designed Primers Sequences for Microcin E492

\begin{tabular}{lll}
\hline Primers Name & Primer Sequence & Length \\
\hline FMN (forward) & 5'-ATCGACGGATCCGGGCGAGACCGATCCAAATAC-3' \\
RMN (reverse) & 5'-CAACATCTCGAGACTACCACTACCGGAACTGGATG-3' & 33 \\
\hline & & 35 \\
\hline
\end{tabular}

Table 2. Conditions for Using Hot Start PCR Reaction

\begin{tabular}{lccc}
\hline Stage & No. of Cycle & Temperature ${ }^{\circ} \mathrm{C}$ & Incubation Time \\
\hline Initial denaturation & 1 & 94 & $5 \mathrm{~min}$ \\
Denaturation & 35 & 94 & $45 \mathrm{sec}$ \\
\hline Annealing & & 64 & $75 \mathrm{sec}$ \\
\hline Extension & & 72 & $30 \mathrm{sec}$ \\
\hline Final extension & 1 & 72 & $5 \mathrm{~min}$ \\
\hline
\end{tabular}

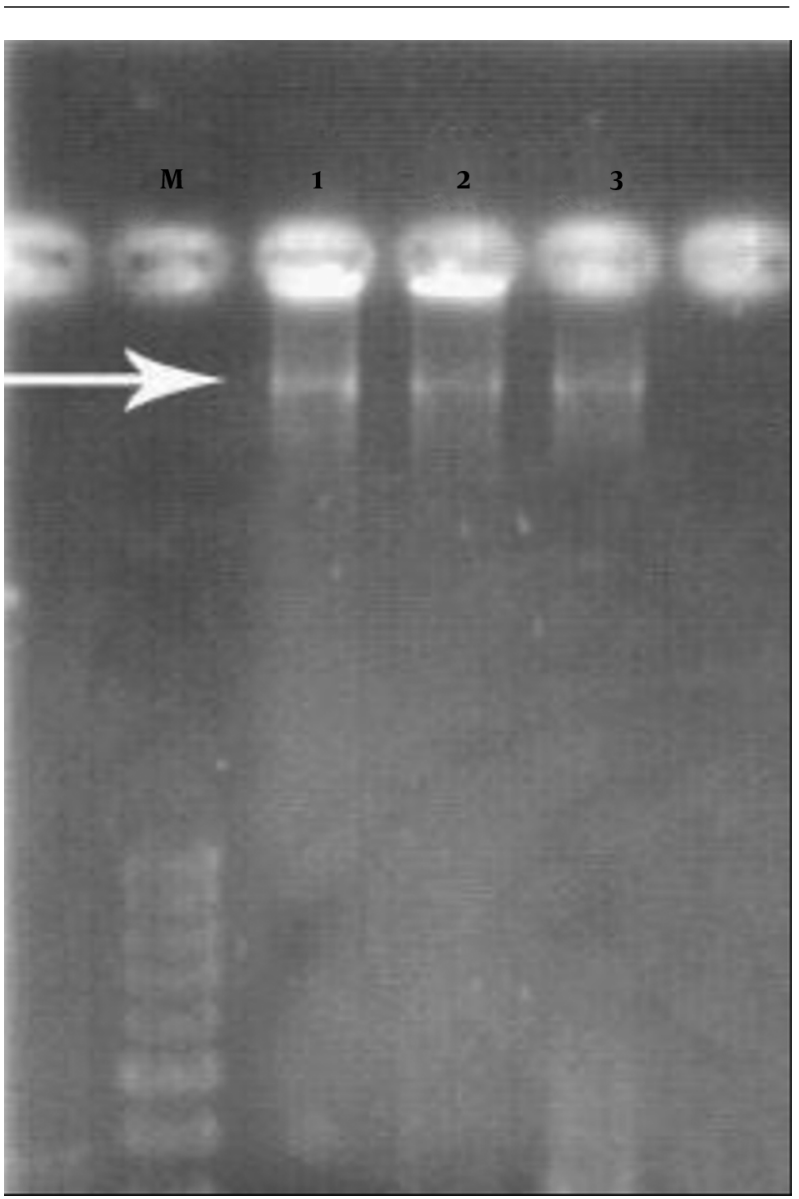

Figure 1. DNA Extracted from Cultured Klebsiella pneumonia, Electrophoresis in $1 \%$ Agarose Gel

\subsection{Statistic of Analysis of Microcin E492 Gene}

This descriptive statistic of analysis showed a notable prevalence of microcin E492 gene in Klebsiella pneumoniae isolated from the patients (Figure 3). 20 clinical specimens of the total of clinical specimens were infected with Klebsiella pneumonia (about 44\%). We detected microcin E492 in about $40 \%$ of $K$. pneumoniae isolates (8 cases of 20 infected cases): 1 (5\%) of the burn wound, 4 (20\%) of urine and $3(15 \%)$ of the isolates were respiratory tracts. Thus, all the strains that had been diagnosed by phenotypic methods were approved.

\section{Discussion}

In this study, microcin E492 gene from Klebsiella pneumoniae was screened by using PCR in 45 clinical specimens of patients. Sequencing confirmed the fidelity of amplicon. Results showed a high prevalence of Klebsiella pneumoniae strains harboring this gene, which can be an explanation of these bacteria being a common microbial flora in some of Iran's hospitals. More experiments and research in the sources of the microorganisms were carried out, Extraction and separation of microcin E492 gene were also carried out by using standard already prepared strains. For example, the study was done by De Lorenzo et al., The effect of microcin produced by Klebsiella pneumoniae on E. coli in a co-culture condition confirmed the sensitivity of Escherichia coli to microcin. They used various culture for microorganisms but prepared microcin by M9 melted soft agar medium, and finally filtered supernatants [18]. In contrast, we used 45 clinical specimens of patients for conventional biochemical and standard microbiology tests in initial identification. Microcin E492 is a low molecular weight antibiotic peptides produced by Enterobacteriaceae with a 
A

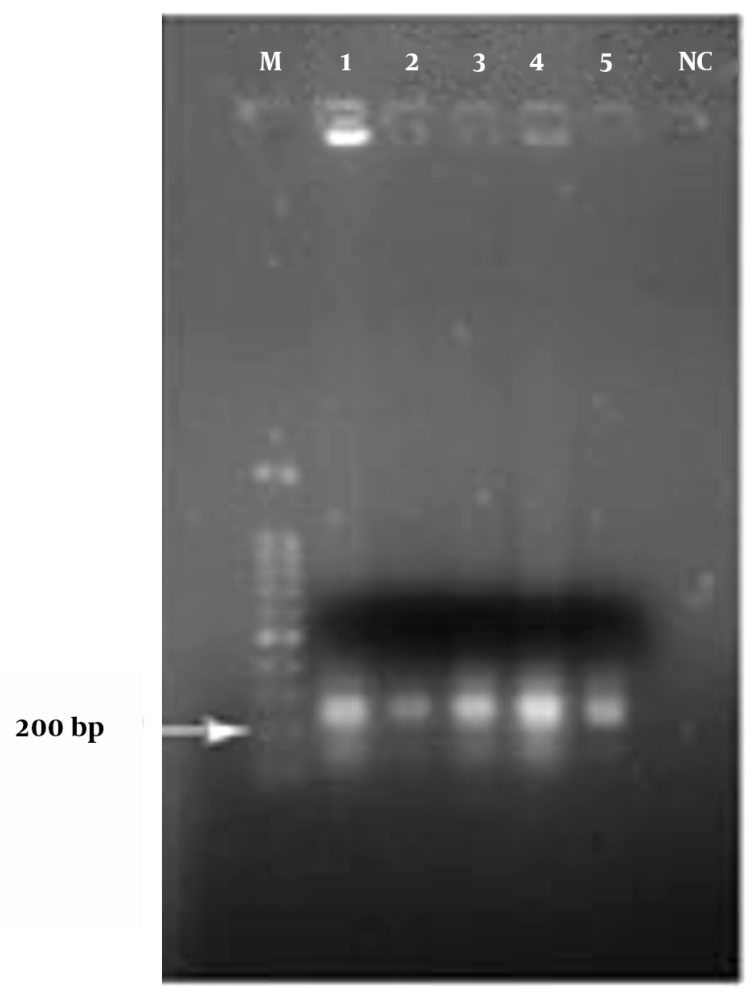

B

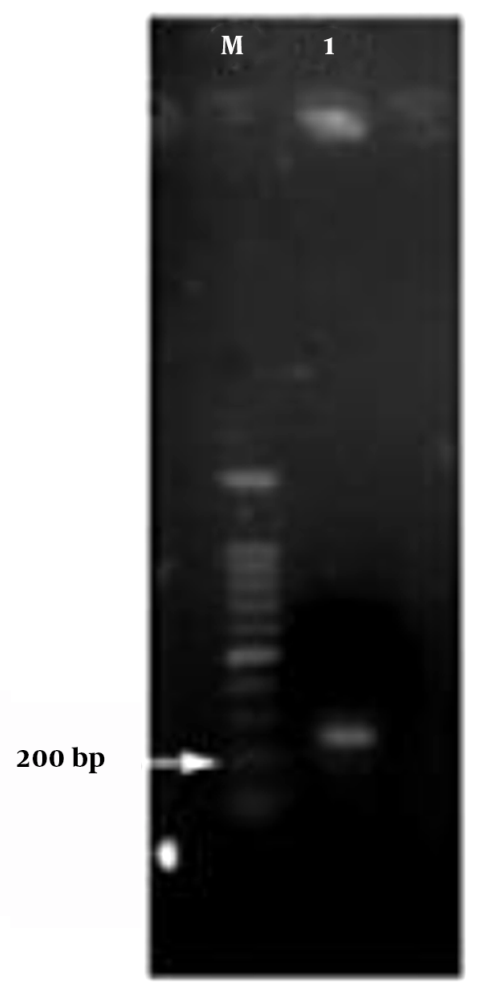

Figure 2. (A) Electrophoresis of PCR products on 1\% agarose gel, Arrow shows band 200 bp and the fragment can be seen on top of it. (1-5: PCR products and M: Marker VC 100 bp Plus and NC, negative control), (B) the PCR product extracted from a 1\% agarose gel. Arrow shows band 200. (M: MarkerVC 100 bp plus, 1: One of the products extracted from the gel)

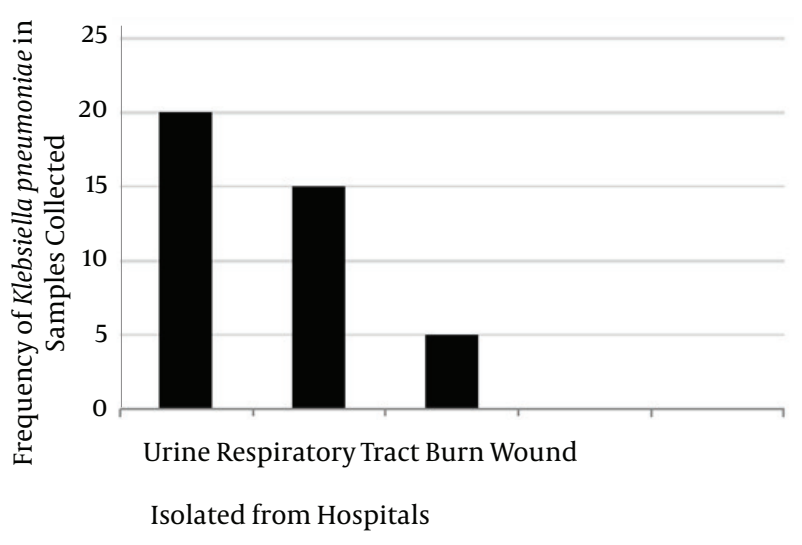

Figure 3. Frequency (\%) Distribution of Microcin E492 in 20 Isolated Klebsiella pneumoniae Isolated from Isfahan Hospitals

wide range of antibacterial activity against Gram-negative bacteria. This property can be useful for antibacterial tri- als.

Hetz et al. (2002) found that microcin E492 has a cytotoxic effect, low molecular weight, and formation of the channel was produced by strains of Klebsiella pneumoniae. Induction of apoptosis by microcin E492 due to the calcium ion released from intracellular stores. Moreover they found out a specific interaction between bacteriocin and the components of the target cell surface. Microcin amino acid sequence analysis showed that microcin E492 belongs to a new class of bacteriocin pore-forming. They extracted and purified microcin E492 from supernatant cultures of E. coli VCS257pJEM15 by M9 medium, and then purified it by HPLC method [19]. Therefore in our study, we extracted microcin E492 from Klebsiella pneumonia directly.

De Lorenzo et al. found out that the pure product characteristics revealed that microcin E492 is a hydrophobic peptide with molecular weight about $5 \mathrm{kD}$, resistant to heat and acid. Microcin E492 is produced and is active in Gramnegative strains: E. coli, Klebsiella, Salmonella, Citrobacter, Enterobacteror erwinia but it is not produced in Shigella, Pro- 
teus, Serratia or Pseudomonas. Furthermore, the sensitivity of some non-agglutinable type strains of Vibrio was observed at high concentrations of microcin. In his study, Klebsiella pneumonia RYC492 was used to detect microcin E492 and HPLC method was used to purify it [20]. Because the majority of studies available, used a limited number of samples, this study cannot be statistically compared with the other research findings.

In this study, because of the high importance of microcin E492 in medicine, we focused on its prevalence in bacteria isolated from hospitals. Past and ongoing researches on antimicrobial peptides have shown that these compounds have a great potential to be used in food and medical industries. Discoveries of new antimicrobial peptides and the understanding of the biological process involved in the synthesis, immunity, and regulation of antimicrobial peptides, should play a role in this field, with emphasis on practical applications in the industry [3]. In this study, we isolated Klebsiella pneumoniae strains from the burn wounds, urine and respiratory tract in more than $40 \%$ of patients' clinical specimens. In the next step, we screened the Microcin E492 in these isolates. Our result can support high prevalence of Klebsiella pneumoniae in hospital infections. In other words, K. pneumoniae can use microcin E492 as a weapon against other microbial flora. As reported in this study, existence of microcin gene in Enterobacteriaceae can develop them to a dominant microbial flora in human body. In addition, based on the results obtained in this study and the problem of resistance to beta-lactam agents and third generation cephalosporins, which are the most effective broad-spectrum antibiotics in the treatment of many infectious diseases, this is a serious problem for the development, and we also need to replace the new antibiotics.

\subsection{Conclusion}

Our results showed that $40 \%$ of patients' clinical specimens were infected with Klebsiella pneumonia, an inevitable outbreak of infections in some hospitals of Iran. High prevalence of microcin gene in these bacteria can result in a diminishing native microbial flora. Since exchange of microcin is in an extra genomic manner, focusing on this phenomenon is a necessary goal of future researches.

\section{Acknowledgments}

This article is statistical part report of a MSc. Thesis by Mrs. Maryam Nasr Esfahani supervised by Dr. Ali Mohammad Ahadi with track number: 1082043 in Irandoc.

\section{Footnotes}

Authors' Contribution: All authors had equal role in design, work, statistical analysis and manuscript writing.

Conflict of Interest: No conflict of interests.

Funding/Support: Falavarjan branch of Islamic Azad University.

\section{References}

1. Nguyen DT, Lessor LE, Cahill JL, Rasche ES, Kuty Everett GF. Complete Genome Sequence of Klebsiella pneumoniae CarbapenemaseProducing K. pneumoniae Siphophage Sushi. Genome Announc. 2015;3(5) doi: 10.1128/genomeA.00994-15. [PubMed: 26337889].

2. Holt KE, Wertheim H, Zadoks RN, Baker S, Whitehouse CA, Dance D, et al. Genomic analysis of diversity, population structure, virulence, and antimicrobial resistance in Klebsiella pneumoniae, an urgent threat to public health. Proc Natl Acad Sci U S A. 2015;112(27):E3574-81. doi: 10.1073/pnas.1501049112. [PubMed: 26100894].

3. Shetty K, Paliyath G, Pometto A, Levin RE. Food biotechnology. second ed ed. CRC Press: Taylor \& francis group; 2005.

4. de Lorenzo V, Pugsley AP. Microcin E492, a low-molecular-weight peptide antibiotic which causes depolarization of the Escherichia coli cytoplasmic membrane. Antimicrob Agents Chemother. 1985;27(4):666-9. [PubMed: 2408563].

5. Gulluce M, Karaday M, Barıs O. . Bacteriocins: Promising Natural Antimicrobials. science, technology and education. 2013 :1016-27.

6. Lagos R, Tello M, Mercado G, Garcia V, Monasterio O. Antibacterial and antitumorigenic properties of microcin E492, a pore-forming bacteriocin. Curr Pharm Biotechnol. 2009;10(1):74-85. [PubMed: 19149591].

7. Arnison PG, Bibb MJ, Bierbaum G, Bowers AA, Bugni TS, Bulaj $G$, et al. Ribosomally synthesized and post-translationally modified peptide natural products: overview and recommendations for a universal nomenclature. Nat Prod Rep. 2013;30(1):108-60. doi: 10.1039/c2np20085f. [PubMed: 23165928].

8. Lagos R, Baeza M, Corsini G, Hetz C, Strahsburger E, Castillo JA, et al. Structure, organization and characterization of the gene cluster involved in the production of microcin E492, a channel-forming bacteriocin. Mol Microbiol. 2001;42(1):229-43. [PubMed: 11679081].

9. Vassiliadis G, Destoumieux-Garzon D, Peduzzi J. In Prokaryotic Antimicrobial Peptides. New York Dordrecht Heidelberg London; 2011. pp. 309-32.

10. Arranz R, Mercado G, Martin-Benito J, Giraldo R, Monasterio O, Lagos $\mathrm{R}$, et al. Structural characterization of microcin E492 amyloid formation: Identification of the precursors. J Struct Biol. 2012;178(1):54-60. doi: 10.1016/j.jsb.2012.02.015. [PubMed: 22420976].

11. Marcoleta A, Marin M, Mercado G, Valpuesta JM, Monasterio O, Lagos R. Microcin e492 amyloid formation is retarded by posttranslational modification. J Bacteriol. 2013;195(17):3995-4004. doi: 10.1128/JB.00564-13. [PubMed: 23836864].

12. Brisse S, Grimont F, Grimont PAD. The genus Klebsiella. Prokaryotes. 2006;6:159-96.

13. Alves MS, Dias RC, de Castro AC, Riley LW, Moreira BM. Identification of clinical isolates of indole-positive and indole-negative Klebsiella spp. J Clin Microbiol. 2006;44(10):3640-6. doi: 10.1128/JCM.00940-06. [PubMed:16928968].

14. Singh M, Kakati B, Agarwal RK, Kotwal A. Detection of Klebsiella pneumoniae carbapenemases (KPCs) among ESBL / MBL producing clinical isolates of Klebsiella pneumonia. Inte J current Microbiol. 2015;4(4):726-31.

15. Golshani Z, Ahadi AM, Sharifzade A. Occurrence of ambler class b metallo- $\beta$-lactamase gene in imipenem-resistant pseudomonas 
aeruginosa strains isolated from clinical clinical specimens s. Zahedan J Res Med Sci. 2014;16(2):6-9.

16. Edgar RC. MUSCLE: multiple sequence alignment with high accuracy and high throughput. Nucleic Acids Res. 2004;32(5):1792-7. doi: 10.1093/nar/gkh340. [PubMed: 15034147].

17. Baghani A, Youssefi M, Safdari H, Teimourpour R, Meshkat Z. Designing and Construction Pcdna3.1 Vector Encoding Cfp10 Gene of Mycobacterium tuberculosis. Jundishapur J Microbiol. 2015;8(10):23560. doi: 10.5812/jjm.23560. [PubMed: 26587210].

18. de Lorenzo V, Martinez JL, Asensio C. Microcin-mediated interac- tions between Klebsiella pneumoniae and Escherichia coli strains J Gen Microbiol. 1984;130(2):391-400. doi: 10.1099/00221287-130-2-391. [PubMed: 6374023].

19. Hetz C, Bono MR, Barros LF, Lagos R. Microcin E492, a channel-forming bacteriocin from Klebsiella pneumoniae, induces apoptosis in some human cell lines. Proc Natl Acad Sci U S A. 2002;99(5):2696-701. doi 10.1073/pnas.052709699. [PubMed: 11880624].

20. de Lorenzo V. Isolation and characterization of microcin E492 from Klebsiella pneumoniae. Arch Microbiol. 1984;139(1):72-5. [PubMed: 6385903]. 